REVUE HYBRIDE DE L'ÉDUCATION

Unir nos voix de chercheurs et de praticiens

\title{
Optimiser le questionnement didactique en science et technologie pour mieux rétroagir et évaluer les élèves
}

\author{
Auteurs \\ Nicole Monney, Université du Québec à Chicoutimi, Canada, \\ nicole1 monney@uqac.ca \\ Christine Couture, Université du Québec à Chicoutimi, Canada, \\ christine.couture@uqac.ca \\ Catherine Duquette, Université du Québec à Chicoutimi, Canada, \\ catherine.duquette@uqac.ca
}

Hans Boulay, Université du Québec à Chicoutimi, Canada, hans.boulay1@uqac.ca 


\section{REVUE HYBRIDE DE L'ÉDUCATION}

\section{Résumé}

Cet article présente les résultats d'une recherche collaborative portant sur les pratiques de rétroactions d'enseignants du primaire durant l'enseignement de la science et technologie. La problématique concerne le défi d'instaurer des pratiques évaluatives qui soutiennent l'apprentissage. L'analyse des données issues de deux entrevues semi-dirigées et de trois rencontres en communauté d'apprentissage dessine le portrait évolutif des enseignantes quant à leur compréhension des démarches et des critères d'évaluation en science et technologie. L'interprétation des données permet de dégager un lien entre le questionnement et la rétroaction sur les apprentissages et de souligner l'intérêt de la recherche collaborative pour faire évoluer les pratiques et les cadres de références des chercheurs en éducation.

Mots-clés :

évaluation formative, rétroaction, science et technologie, démarches d'investigation, questionnement 


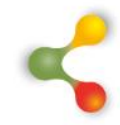

\section{REVUE HYBRIDE DE L'ÉDUCATION}

\section{L'évaluation des apprentissages au Québec et les visées de réussite éducative}

Depuis quelques années au Québec et ailleurs dans le monde, l'intention des politiques éducatives est d'offrir les mêmes chances à tous les élèves (Akkari et Barry, 2018). L'éducation devient un droit fondamental pour chaque élève dont la visée principale est la réussite éducative (Conseil supérieur de l'éducation, 2017; Ramel et Vienneau, 2016; UNESCO, 2016). Dans ce contexte, la question de l'évaluation des apprentissages est souvent pointée du doigt comme un obstacle à la réussite des élèves (Hadji, 2019). La résistance des acteurs de l'éducation et les pressions sociales provenant des parents et de la société en général réduisent l'évaluation des apprentissages à un processus qui aboutit à un résultat chiffré en pourcentage ${ }^{1}$. Le retour du bulletin unique en 2011 en est un exemple (Morrissette et Legendre, 2011). Cette résistance n'est pas étonnante, car comme l'a mis en évidence Bugnard (2004), d'un point de vue historique, la note à l'école permettait de sanctionner les comportements de l'élève. En d'autres termes, l'enseignant détient un pouvoir important sur l'élève et la note qu'il lui donne fait office de motivation extrinsèque. D'autant plus que, bien souvent, la note est le seul référent pour comprendre la progression de l'élève pour le parent. Le fait qu'il ait lui aussi été évalué par le biais d'une note lui permet d'avoir un comparatif. Cependant, la note ne favorise pas l'apprentissage et la conception de l'éducabilité de chaque élève (Prud'homme, Vienneau, Ramel et Rousseau, 2011). L'évaluation dite "formelle», sous forme d'examens, a plutôt tendance à provoquer chez l'élève de l'anxiété, une impression d'incompétence et un désengagement vis-à-vis de l'école (Issaieva et Crahay, 2010). À l'instar de Bugnard (2004), Hadji (2015) constate également que la note est un point de repère commode qui permet des comparaisons faciles et qui peuvent devenir stigmatisantes. Elle sert surtout au classement des élèves et provoque une compétition entre les élèves. De plus, ce type d'évaluation se concentre trop souvent sur les apprentissages académiques qui ne sont qu'une partie de la mission de l'école qui est d'instruire, de socialiser et de qualifier (MEQ, 2001).

Depuis les années 60, plusieurs acteurs de l'éducation militent pour une évaluation qui accompagne l'apprentissage de l'élève en offrant des informations à l'enseignant et à l'élève sur sa progression (Scriven, 1967). Qu'il s'agisse de l'évaluation dite formative (Allal et Lopez, 2007; Morrissette, 2009a; Scallon, 1986; Scriven, 1967), de l'évaluation dite humaniste (Blanvillain et Travnjak, 2019; Eid, 2019; Hadji, 2019) ou, encore, de l'évaluation pour aider l'apprentissage (MEQ, 2003). Ces différents vocables rejoignent tous l'idée que l'évaluation doit avant tout permettre de collecter de l'information sur la progression de l'élève pour remédier à l'enseignement donné et pour permettre à l'élève de réguler ses

\footnotetext{
${ }^{1}$ https://www.ledevoir.com/societe/education/287640/education-quelle-note-pour-le-bulletinunique
} 


\section{$\&$}

\section{REVUE HYBRIDE DE L'ÉDUCATION}

stratégies cognitives et métacognitives. Ce type d'évaluation considère les erreurs de l'élève comme une opportunité de mieux comprendre ses difficultés et pour mieux le soutenir. II ne s'agit ainsi plus de sanctionner l'élève par un résultat chiffré, mais plutôt de situer l'élève dans sa progression au moyen de critères descriptifs ou, encore, dans une démarche herméneutique (De Ketele, 2010). Dans ce contexte, l'évaluation formative est considérée comme un levier à la réussite éducative puisqu'elle n'intègre pas la notion d'échec (Allal et Lopez, 2007; Black et Wiliam, 1998; Deaudelin et al., 2007 ; Hadji, 2015 ; Morrissette, 2009b ; Scallon, 2004).

\section{Le défi d'adopter des pratiques évaluatives qui soutiennent l'apprentissage}

Bien que l'évaluation formative soit reconnue comme un processus qui favorise la progression de l'élève dans ses apprentissages, les pratiques évaluatives à mettre en place restent un défi pour les enseignants. Selon Harlen (2016), la façon dont l'enseignant définit l'apprentissage influence sa façon d'évaluer ses élèves. Depuis 2011, les enseignants n'ont pas le choix de mettre une note en pourcentage pour chacune des disciplines scolaires. Par conséquent, l'apprentissage peut être vu comme un processus sommatif d'un ensemble de connaissances acquises. Dans ce contexte, les enseignants auraient tendance à prioriser des pratiques évaluatives qui sanctionnent la progression de l'élève (Laurier, 2014). Par ailleurs, la pression de réussite aux examens du Ministère favorise également des pratiques dites «teach to the test» (Laveault, 2014). L'enseignant considère l'apprentissage comme un moment de préparation à l'examen qui, souvent, vise à mesurer l'acquisition de connaissances (Monney, Duquette, Couture, Boulay, sous presse). Les résultats à ces examens sont souvent utilisés pour réaliser le palmarès des écoles chaque automne. Les directions d'école s'en servent pour évaluer la performance des enseignants. II est donc difficile pour un enseignant d'ignorer la pression amenée par la réussite à ces épreuves et ne pas entrer dans un processus de « teach to the test ».

Afin de réfléchir à cette problématique, trois chercheures issues d'expertises différentes (didactique de la science et de la technologie, didactique de l'univers social, évaluation des apprentissages) ont mis sur pied un projet de recherche collaborative avec des enseignantes du primaire dont les objectifs étaient d'identifier les objets d'apprentissages ciblés par les enseignantes et de dégager les rétroactions qu'elles faisaient aux élèves par rapport aux objets d'apprentissage enseignés. ${ }^{2}$ Cet article présente une partie des résultats de la recherche, à savoir l'arrimage entre les gestes didactiques en science et technologie et les gestes évaluatifs des enseignants. Le contexte de la recherche collaborative a permis aux

\footnotetext{
${ }^{2}$ Conseil de recherche en sciences humaines du Canada : Subvention développement savoir (430-2017-00344)
} 


\section{$\&$}

\section{REVUE HYBRIDE DE L'ÉDUCATION}

chercheures de prendre conscience des possibilités pour la rétroaction en évaluation des apprentissages qu'offre le questionnement en didactique de la science et de la technologie.

\section{Cadre conceptuel : l'arrimage entre l'activité didactique et l'évaluation des apprentissages}

Black (2016) a mis en évidence que la réflexion autour de l'évaluation formative devait s'inscrire dans l'activité didactique. Hasni, Bousadra et Marcos (2011) ont constaté que les savoirs ciblés en science et technologie privilégiaient souvent le français et les mathématiques, et ce, au détriment des savoirs scientifiques qui arrivent parfois au second plan. II importe ainsi de clarifier quels sont les objets d'apprentissage en science et technologie et quelles sont les démarches favorisées en didactique des sciences et des technologies pour bien arrimer l'apprentissage et l'évaluation et par conséquent, mieux rétroagir auprès des élèves.

\section{Les objets d'apprentissages en science et technologie}

Les objets d'apprentissages en science et technologie sont identifiés dans le programme de formation de l'école québécoise (MELS, 2009, 2011; MEQ, 2001) et se déclinent en termes de connaissances, de démarches, de stratégies, de techniques et d'habiletés. Ces objets devraient constituer la base sur laquelle l'enseignant planifie ses activités d'enseignement et devraient être liés aux critères d'évaluation proposés par les cadres d'évaluation (MELS, 2011). Notons que pour le volet science et technologie du programme, les critères se présentent comme suit: description adéquate du problème, mise en œuvre d'une démarche appropriée, utilisation appropriée d'instruments, d'outils et de techniques et utilisation appropriée des connaissances scientifiques et technologiques (MELS, 2009). Par conséquent, si un enseignant aborde les états de la matière dans une activité de science, il pourrait évaluer ces connaissances en essayant de voir comment les élèves les mobilisent pour expliquer le cycle de l'eau à l'aide du critère d'évaluation "utilisation appropriée des connaissances scientifiques et technologiques ". Le but n'est alors pas d'évaluer si l'élève a retenu des définitions relatives aux états et à la transformation de la matière, mais plutôt d'observer comment l'élève utilise ces connaissances pour produire des explications ou des solutions à un problème d'ordre scientifique ou technologique. L'avantage de cette approche est que l'enseignant peut rétroagir pendant l'activité sur la façon de réutiliser les connaissances dans différents contextes pour formuler des explications.

\section{Les démarches en didactique de la science et technologie}

En didactique de la science et technologie, les démarches menant à la construction des savoirs sont aussi importantes que les savoirs eux- 


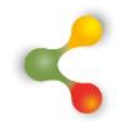

\section{REVUE HYBRIDE DE L'ÉDUCATION}

mêmes (Astolfi, Peterfalvi et Vérin, 1998 ; Coquidé, Fortin et Rumelhard, 2009 ; Lederman et Lederman, 2012). Cette complémentarité entre les démarches mises en œuvre pour la construction des savoirs est d'ailleurs au cœur des compétences à développer dans le programme et se reflète à travers les critères proposés dans le cadre d'évaluation des apprentissages (MELS, 2011). La première compétence du programme en science et technologie (MELS, 2001), qui consiste à " proposer des solutions et des explications à des problèmes d'ordre scientifique et technologique ", ainsi que la deuxième qui incite à «mettre à profit les outils, objets et procédés de la science et de la technologie ", sont liées aux critères (MELS 2009) relatifs à « la description adéquate du problème », " la mise en œuvre d'une démarche appropriée » ainsi qu'à " l'utilisation appropriée d'instruments, d'outils et de techniques ". La troisième compétence qui vise à "communiquer à l'aide des langages utilisés en science et technologie " est en lien avec " l'utilisation appropriée des connaissances scientifiques et technologiques " sans que la maitrise des connaissances se limite à cette troisième compétence. Cette mise en relation des compétences visées et des critères d'évaluation souligne l'importance des démarches proposées dans le programme (MELS 2001), dont le questionnement, l'observation méthodique, le tâtonnement, la vérification expérimentale, l'étude des besoins et des contraintes, la conception de modèles et la réalisation de prototypes. C'est à travers ces démarches que devraient être abordés les savoirs et les stratégies proposés dans la progression des apprentissages (MELS, 2009). Le questionnement étant au cœur de l'activité scientifique, il traverse toutes ces démarches et se manifeste à toutes les phases de réalisation d'une activité de science et technologie. II importe donc de questionner l'élève tout au long de la démarche pour travailler dans le sens des compétences et en saisir des manifestations en lien avec les critères d'évaluation. II est par conséquent intéressant de regarder les dimensions mobilisées par les questions posées par les enseignants pour accompagner leurs élèves dans l'intention de réfléchir à la rétroaction et à l'évaluation.

\section{La rétroaction en évaluation des apprentissages}

La rétroaction s'inscrit dans la fonction de régulation de l'évaluation des apprentissages (De Ketele, 2010). La rétroaction se définit comme une information donnée par l'enseignant ou un pair sur la compréhension relative aux objets d'apprentissage abordés (Hattie et Timperley, 2007), et ce, pendant une activité ou après celle-ci. Elle peut se faire de façon informelle par le biais de commentaires faits à l'oral ou encore, de façon formelle par le biais de commentaires écrits à partir d'instruments d'évaluation (grille d'évaluation, commentaires dans une production de l'élève, etc.) (Diedhiou, 2013; Jorro et Mercier-Brunel, 2011; Talbot et Arrieu-Mutel, 2012).

Pour être efficaces, les rétroactions doivent être qualitatives, c'est-àdire qu'elles doivent décrire les lacunes et les forces de l'élève et proposer 


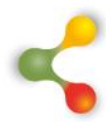

\section{REVUE HYBRIDE DE L'ÉDUCATION}

des stratégies de remédiation (Hattie et Timperley, 2007). La note n'est donc pas considérée comme une rétroaction. Les rétroactions portent sur quatre catégories d'éléments : la tâche, le processus durant la tâche, l'autorégulation et l'autoévaluation et la personne (Hattie et Timperley, 2007). La rétroaction sur la tâche consiste à donner à l'élève une rétroaction pour assurer la compréhension de la tâche. En science et technologie, l'enseignant pourrait demander à l'élève d'expliquer ce qu'il comprend d'une observation, d'une expérience ou d'un modèle, en lien avec les concepts. La rétroaction sur le processus durant la tâche concerne des rétroactions qui portent sur le déroulement de l'activité, en lien avec les démarches d'investigation. L'enseignant pourrait ainsi prendre un temps pour réfléchir, avec l'élève, sur l'élaboration ou la réalisation d'un protocole pour répondre à une question ou sur les choix possibles lors de la conception d'un modèle ou d'un prototype. La rétroaction sur l'autorégulation et l'autoévaluation vise à faire prendre conscience à l'élève des stratégies qu'il met en place. L'enseignant pourrait demander à l'élève de revoir sa démarche, de la comparer avec celle d'un autre élève et de l'ajuster au besoin. Finalement, la rétroaction sur la personne consiste à féliciter l'élève pour ses qualités personnelles ou, encore, à identifier ses défis. Ce type de rétroaction ne porte pas sur les objets d'apprentissage, mais plutôt sur la personne elle-même.

La rétroaction doit mener à la régulation des stratégies cognitives et métacognitives de l'élève (Allal et Lopez, 2007), ce qui permet de faire le lien avec les stratégies d'exploration pour déployer les démarches d'investigation en science et technologie. Par conséquent, elle doit nécessairement s'appuyer sur les objets et les démarches d'apprentissage ciblés par une activité (Mottier Lopez, 2015). Aussi, dans un souci de cohérence, les rétroactions de l'enseignant devraient se faire à partir des critères d'évaluation présentés plus haut.

La figure 1 représente l'arrimage entre les éléments du cadre conceptuel, à savoir la didactique de la science et technologie et l'évaluation des apprentissages. 


\section{$\&$}

\section{REVUE HYBRIDE DE L'ÉDUCATION}

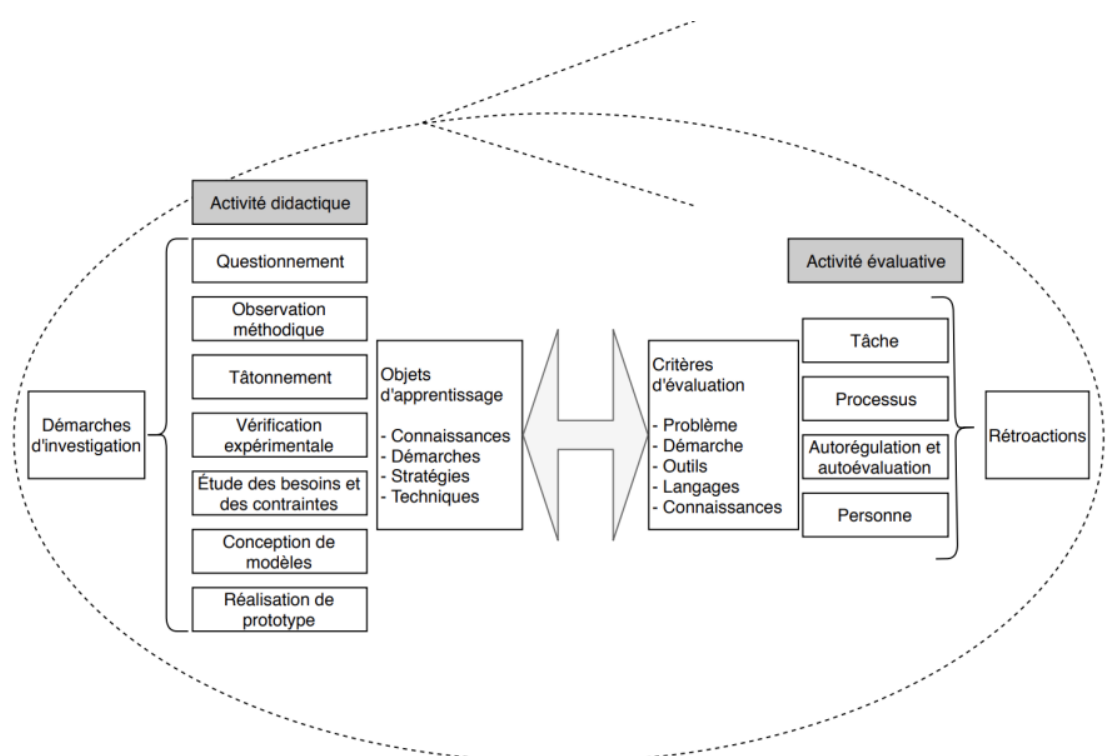

Figure 1 : Arrimage des concepts issus de la didactique de la science et technologie et de l'évaluation des apprentissages

La figure intègre à la fois les éléments en lien avec l'activité didactique et l'activité évaluative. Ainsi, dans l'activité didactique, on retrouve les démarches d'investigation et les objets d'apprentissage. Du côté de l'activité de l'évaluation, les rétroactions se font sur différents niveaux, et ce, en regard des critères d'évaluation. Finalement, les flèches au centre signifient que les rétroactions ne peuvent se faire sans prendre en compte les objets d'apprentissages. La flèche ovale entourant l'ensemble des concepts rappelle que l'activité didactique et l'activité évaluative sont interreliées. L'analyse des données s'est ainsi réalisée en s'appuyant sur cette figure.

\section{Méthodologie : la recherche collaborative pour coconstruire autour des pratiques de rétroactions}

Les chercheures s'inscrivent dans une approche constructiviste de la recherche dans l'idée que les connaissances issues de cette recherche sont construites et orientées vers des finalités, dépendantes des actions et des expériences faites par les acteurs dans leur contexte particulier (Mucchielli, 2005). Pour opérationnaliser la recherche, les chercheures ont opté pour le modèle de la recherche collaborative (Desgagné, 1997). Ce modèle demande de prendre en compte le point de vue des enseignants en favorisant le maillage d'expertises des praticiens et des chercheurs (Bednarz, 2013). Elle a pour caractéristiques de produire des résultats qui présentent une double vraisemblance des résultats (Dubet, 1994) pour la recherche et pour la pratique. La recherche collaborative comprend trois étapes essentielles: la cosituation, la coopération et la coproduction 


\section{$\&$}

\section{REVUE HYBRIDE DE L'ÉDUCATION}

(Desgagné, 1997). Le chercheur doit donc respecter et refléter, aux différentes étapes de la recherche, la logique de deux mondes, soit celui de la recherche et celui de la pratique (Barry et Saboya, 2015).

La recherche a commencé en décembre 2017 dès l'obtention de la certification éthique de la part de l'université d'attache (CE-602-184-07) et a duré deux ans. Les participantes étaient sept enseignantes du primaire provenant d'écoles différentes dans une même commission scolaire. Plus précisément, deux enseignantes étaient titulaires d'une classe de $6^{\mathrm{e}}$ année primaire $(E 3 ; E 4)$ et trois enseignantes étaient en $5^{\mathrm{e}}$ année primaire (E2, $E 5$, E6). Les deux dernières enseignantes étaient en $2^{\mathrm{e}}$ année primaire. Cependant, comme l'évaluation de la science et technologie n'est pas prescrite officiellement au premier cycle du primaire, elles se servaient de l'enseignement de la science et de la technologie pour rétroagir sur des objets d'apprentissage en français et en mathématique. C'est pourquoi, pour cet article, seules les données provenant des enseignantes du $3^{\mathrm{e}}$ cycle sont présentées et analysées.

\section{Déroulement de la collecte de données}

Le devis méthodologique suit les étapes de la recherche collaborative et intègre des journées de réflexion en équipe et des entrevues semi-dirigées. Le tableau 1 présente le déroulement de la recherche ainsi que les méthodes de collecte de données.

Tableau 1 : Devis méthodologique

\begin{tabular}{lll} 
Étapes & Dates & Collecte de données \\
\hline Cosituation & Déc. 2017 & Rencontre et focus group (6 heures) \\
Coopération & Février 2018 & Entrevue semi-dirigée (60 - 75 min.) \\
Coopération & Mars 2018 & Rencontre et focus group (6 heures) \\
Coopération & Avril 2018 & Entrevue semi-dirigée (60-75 min.) \\
Coproduction & Oct. 2018 & Rencontre et focus group (6 heures) \\
Coproduction & Déc. 2018 & Rencontre et focus group (6 heures) \\
Coproduction & Mai 2019 & Rencontre et focus group (6 heures)
\end{tabular}

La rencontre de décembre 2017, a permis de négocier l'orientation de la recherche avec les enseignants et, particulièrement, sur les besoins de stratégies pour poser un jugement sur la progression de l'élève et pour mettre une note représentative de cette progression. Les données qui concernent plus spécifiquement les rétroactions des enseignants en enseignement de la science et technologie ont été collectées durant la première entrevue semi-dirigée en février 2018, le focus group de mars 2018, l'entrevue semi-dirigée d'avril 2018 et finalement, le focus group de décembre 2018. Ainsi, les résultats présentés dans cet article sont des 


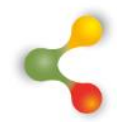

\section{REVUE HYBRIDE DE L'ÉDUCATION}

données qualitatives issues d'entrevues semi-dirigées ainsi que de focus group.

En février 2018, une première entrevue semi-dirigée d'une durée de 60 à 75 minutes a été menée auprès de chaque enseignante. Le canevas d'entrevue avec quelques questions ouvertes comprenait deux axes: les objets d'apprentissages ciblés et l'évaluation des apprentissages. Le premier axe comprenait sept questions qui abordaient les objets d'apprentissage ciblés, le choix de ces objets d'apprentissage, le temps de planification, la façon de planifier (en équipe, les éléments importants), les outils utilisés pour planifier, les priorités et les réalisations des élèves. L'axe deux comprenait cinq questions à savoir, les pratiques d'évaluation, la fonction de l'évaluation, les outils pour évaluer, les pratiques de rétroactions et les pratiques de planification de l'évaluation. Ces questions permettaient de dégager les pratiques déclarées par les enseignants pour cibler les apprentissages et pour rétroagir auprès de leurs élèves.

En mars 2018, soit un mois après les entrevues, les chercheures et les praticiennes se sont rencontrées pour porter un regard analytique sur une première synthèse du contenu des entrevues semi-dirigées. II s'agissait de trianguler les observations des chercheures avec le regard des enseignantes. Étant en recherche collaborative, les données se sont construites en collaboration, Les enseignantes ont pris connaissance des stratégies d'évaluation, des objets d'évaluation et des défis à relever en évaluation. Elles ont commenté très librement les différents résultats présentés par les chercheures. La journée a permis d'échanger autour des critères d'évaluation en science et technologie et de mettre de l'avant l'interprétation que chacun en faisait. La question des types de rétroactions qu'on peut faire a été abordée brièvement. Finalement, les enseignantes sont reparties avec un tableau dans lequel elles devaient intégrer les critères d'évaluation qu'elles ciblaient, décrire la situation d'apprentissage, identifier les questions posées aux élèves et identifier les réussites et les blocages que les élèves vivaient.

En avril 2018, la deuxième entrevue semi-dirigée visait à échanger autour du tableau remis lors de la dernière journée de réflexion. L'idée de cette entrevue était d'approfondir la compréhension autour des pratiques de rétroactions et de dégager comment les difficultés des élèves étaient surmontées par les enseignants. Le deuxième axe portait sur la rétroaction et comptait quatre questions : le moment des rétroactions, les cibles de la rétroaction, l'adaptation de la rétroaction par rapport à un élève, et l'objet sur lequel porte la rétroaction. Le dernier axe portait sur la planification des objets d'apprentissages et compte six questions : l'objet d'apprentissage, la cible d'apprentissage, le processus de l'activité, la production finale de l'élève, la planification de la situation et les pratiques d'évaluation. Le but étant d'approfondir les pratiques de rétroactions des enseignantes. 


\section{$\&$}

\section{REVUE HYBRIDE DE L'ÉDUCATION}

En décembre 2018, soit huit mois après la deuxième entrevue, les chercheures et les praticiennes se sont rencontrées pour mener une analyse sur les ajustements de pratiques apportés par les enseignants à propos de la rétroaction. Les chercheures ressentaient le besoin de revenir sur les types de rétroactions et de proposer une réflexion sur les démarches en science et technologie. Les enseignantes ont donc commenté un tableau comprenant les différents types de rétroactions qu'elles avaient proposés durant la deuxième entrevue. Elles ont également réfléchi à des questions pertinentes pouvant être posées aux élèves pour les accompagner dans leur progression.

\section{Analyse des données}

L'analyse des données issues des focus groups et des entrevues suit les phases proposées par L'Écuyer (1990). La première étape consiste à coder les données transcrites sur le logiciel NVivo en partant des catégories proposées par le cadre conceptuel. Ce codage permet de découper les propos des enseignantes en séquences catégorisées comme : rétroaction, objet d'apprentissage, démarche, stratégie, etc. La première analyse faite sur ce codage est de type délibéré. La deuxième étape consiste à reprendre les séquences codées et à identifier des catégories émergentes. Cette analyse est donc inductive et permet de réaliser un codage mixte (Van der Maren, 1995). Ainsi, la première étape de l'analyse a permis d'identifier les objets d'apprentissage et de dégager les rétroactions faites par les enseignants. La deuxième étape de l'analyse a mis en évidence le lien qui existe entre certains gestes didactiques, comme le questionnement, et les pratiques de rétroaction des enseignants.

\section{Résultats}

Les résultats présentés dans ce texte mettent en lumière le chemin parcouru par des enseignants quant à leur compréhension des objets d'apprentissage à évaluer en science et technologie. La présentation se réalise selon l'ordre chronologique de la collecte de données à savoir : la première entrevue, le focus group de mars, la deuxième entrevue, le focus group du mois d'avril 2018 et le focus group du mois de décembre 2018.

\section{Première entrevue (février 2018): le choix des objets d'apprentissage et les pratiques de rétroactions des enseignantes}

Le premier constat réalisé après les premières entrevues était celui de la compréhension des démarches d'investigation en science et technologie. Les exemples suivants permettent d'illustrer ce constat. 


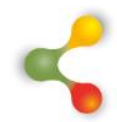

\section{REVUE HYBRIDE DE L'ÉDUCATION}

E2, enseignante en $6^{\mathrm{e}}$ année du primaire, présente l'activité de la valise 00 watt $^{3}$. Selon la trousse, l'intention des activités proposées dans cette trousse pédagogique est de sensibiliser les élèves à la nécessité d'économiser l'électricité à travers des activités et des manipulations. E2 mentionne suivre le guide pour identifier les objets d'apprentissage. Les élèves ont à lire des textes, faire des jeux en ligne et réaliser des circuits électriques. Pour évaluer, E2 cible le critère de la mise œuvre d'une démarche appropriée ainsi que celui de l'utilisation appropriée des connaissances scientifiques et technologiques. Voici un extrait qui illustre sa compréhension de la mise en place d'une démarche appropriée :

"Mise en place d'une démarche appropriée, il faut qu'il soit autonome. S'il y a une équipe, on leur dit 4 fois, va relire le protocole, parce qu'ils ne l'ont pas lu, ça nous indique que ce n'est pas correct, je vais aller les questionner. " (E2, entrevue 1, février 2018)

Cet exemple met en évidence que le critère est interprété comme l'habileté à suivre un protocole. Le but, dans cet exemple, n'est pas de créer une démarche pour répondre à une question d'ordre scientifique comme proposé dans le PFEQ (MEQ, 2001). Bien que la rétroaction porte sur le processus de la tâche, elle ne porte pas sur la mise en œuvre de la démarche appropriée, mais plutôt sur les stratégies de lecture de l'élève. Elle n'est ainsi pas nécessairement centrée sur les démarches d'investigation en science et technologie, mais plutôt sur des démarches qui pourraient être reliées au français, par exemple. Le deuxième extrait amène le même constat sur le deuxième critère qui est l'utilisation appropriée d'instruments, d'outils et de techniques :

\begin{abstract}
"Quand on a fait l'acide des bases, j'étais assise à la table du matériel et je ne vérifiais que la mesure des élèves. Est-cke qu'ils s'installent sur la table? Ont-ils une technique? Ma collègue vérifiait s'ils suivaient un protocole, s'ils ont bien lu les étapes. " (E2, entrevue 1, février 2018)
\end{abstract}

Ainsi, E2 vérifie la technique des élèves pour mesurer des liquides et, ce qu'elle évalue, c'est la justesse de la mesure. Dans une démarche didactique, l'idée serait plutôt de questionner l'élève sur la raison de mesurer correctement un liquide, d'observer ce qui se passe, de dégager les erreurs ou, encore, de réfléchir aux variables en présence. Dans ce cas, l'accent est mis sur le respect des procédures et du protocole sans nécessairement amener une réflexion autour de l'importance d'utiliser de façon appropriée les instruments, les outils et les techniques.

${ }^{3}$ http://www.hydroquebec.com/professeurs/pdf/valise00watt/guide-enseignement-fr.pdf 


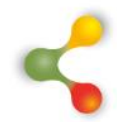

\section{REVUE HYBRIDE DE L'ÉDUCATION}

E5, enseignante en $5^{e}$ année du primaire, a proposé une activité nommée "Alibobo, les meilleurs pansements ». Cette activité a pour but d'évaluer la capacité des élèves à mettre en œuvre une démarche appropriée pour vérifier la qualité de différents pansements. Chaque équipe se voit assigner une propriété à vérifier. Pour ce faire, les élèves élaborent leur propre démarche expérimentale, ils la mettent à l'essai et ils l'ajustent au besoin. E5 a mentionné évaluer le critère de mise en place d'une démarche appropriée. L'analyse de l'entrevue a mis en évidence que cet exemple se rapprochait davantage du critère d'évaluation portant sur la démarche.

\section{Focus group (mars 2018) : rétroagir à partir des critères d'évaluation}

Lors de la deuxième rencontre, les échanges ont porté autour des critères d'évaluation pour comprendre comment les enseignantes structuraient leurs activités à partir de ceux-ci et quelles étaient les rétroactions réalisées auprès des élèves.

E3 mentionne utiliser les critères d'évaluation pour bien expliquer aux élèves ce qu'elle attend d'eux. Son attention porte surtout sur la justesse du vocabulaire utilisé par l'élève.

\begin{abstract}
"(à partir de la grille d'évaluation). J'étais capable d'expliquer à mes élèves quoi faire pour avoir telle note. Je trouvais que quand j'arrivais pour l'hypothèse, plus tu prends les vrais termes et plus tu détailles, tu vas avoir plus de points que quelqu'un qui fait une hypothèse très courte de niveau de $3^{\ominus}$ année. Le jeune le sait ce que j'attends de lui pour avoir telle note [...] Ils peuvent savoir pourquoi ils ont ça. Là ils sont capables de dire qu'ils auraient dû dire le mot " trombone " au lieu de dire "cet objet ". Ça fait partie des connaissances et de prendre les bons termes en sciences. " (E3, Focus group, mars 2018)
\end{abstract}

Cet extrait illustre l'importance de la note dans la démarche de l'enseignante. Le but annoncé aux élèves est d'avoir une bonne note. Bien qu'elle utilise les critères pour leur expliquer ce qu'elle attend d'eux, l'interprétation qu'elle en fait se centre sur l'hypothèse ou sur la justesse du vocabulaire utilisé. La logique derrière l'évaluation est surtout de vérifier des termes en les comptabilisant pour en arriver à un résultat. Ainsi, l'idée n'est pas d'accompagner l'élève dans l'utilisation appropriée des connaissances scientifiques et technologiques, mais plutôt de sanctionner le choix d'un vocabulaire. 


\section{$\&$}

\section{REVUE HYBRIDE DE L'ÉDUCATION}

Plusieurs échanges ont eu lieu autour des démarches pertinentes en science et technologie. Ainsi, la réflexion a porté autour de la problématisation. Les enseignantes ont soulevé la difficulté à circonscrire un problème scientifique et à en arriver à une question de recherche. E3 propose d'ailleurs un élément de réponse à la difficulté des enseignantes d'accompagner l'élève dans la problématisation.

"Quand on achète des cahiers, on commence et on la donne la question. Nous, on est restée dans cette démarche-là et c'est difficile de changer! » (E3, Focus group, mars 2018)

Au terme de cette journée, il a été possible de constater que même si les critères d'évaluation étaient simples, pour les enseignants, leur interprétation et leur mise en œuvre sont complexes. Aussi, il semblerait que les enseignants restent dans les processus et ont plus de difficulté à dégager les concepts sous-jacents aux activités qu'elles réalisent en science et technologie.

\section{Deuxième entrevue (avril 2018) : mieux cibler ses apprentissages pour mieux rétroagir}

Lors de la deuxième entrevue, les enseignantes ont proposé des rétroactions qu'elles avaient mises en place durant leurs activités. Par ailleurs, il a été possible de constater que les rétroactions étaient plus ciblées et plus centrées sur la tâche et le processus en science et technologie.

E2 a choisi comme activité le défi apprenti génie ${ }^{4}$ en science et technologie. L'activité consiste à créer des avions en papier qui doivent répondre à six différents défis (distance, cible, zone, etc.). Les élèves prennent en note leur démarche de conception et doivent compléter un tableau en indiquant les ajustements techniques. E2 identifie la notion de gravité et l'aérodynamisme comme objet d'apprentissage. Alors que dans la première entrevue, la précision était très importante et évaluée, E2 a fortement réduit la pondération pour ce critère. A la place, elle a opté pour la réalisation d'une carte conceptuelle qui lui permettait d'évaluer le critère d'utilisation appropriée des connaissances scientifiques et technologiques. Elle a également pris le temps d'évaluer le critère de mise en œuvre d'une démarche appropriée. Dans ce contexte, elle a apporté beaucoup de rétroactions à ses élèves sur leur démarche. L'analyse de ces rétroactions met en évidence un changement dans sa compréhension de la démarche en science et technologie. Voici un exemple de question qu'elle a utilisée

\footnotetext{
${ }^{4}$ https://technoscience.ca/programmes/defi-apprenti-genie/
} 


\section{REVUE HYBRIDE DE L'ÉDUCATION}

en guise de rétroactions pour que les élèves autoévaluent et autorégulent leurs démarches:

« Entre autres, il y avait une épreuve où l'avion devait effectuer un virage. La question qu'on a posée [...] * Qu'est-ce que vous avez appris dans les activités préparatoires qui pourraient t'aider à solutionner ton problème? "Des fois juste pour favoriser le lien qui pourrait les aider! Avec tâtonnement, essais et erreurs, retour dans le cahier, ils ont réussi [...] Ils se demandaient quoi aller voir, quelle activité. Ils allaient fouiller et ils se disaient qu'ils pouvaient peut-être ajuster l'aile, ajuster la section du centre... c'est ça. » (E2, entrevue 2, avril 2018)

Dans cet extrait, la rétroaction vise à aider l'élève à autoévaluer sa démarche et à trouver sa démarche pour relever le défi. II n'est plus question de trouver la bonne réponse, mais plutôt de s'appuyer sur les connaissances scientifiques qu'on possède déjà pour réfléchir à un problème et identifier des solutions. Le même constat a pu être fait à partir du discours de E5. Les rétroactions se déroulent dans le cadre d'activités réalisées en robotique où les élèves devaient relever certains défis :

« Si, j'ai une équipe qui est venue me voir et quand ils l'ont fait le capteur était tout mou, quand ça a cogné le capteur n'a jamais eu la pression nécessaire. Moi, quand j'ai remarqué ça tout de suite, la question que j'ai posée aux élèves c'est : "Qu'est-ce que vous pensez qui n'a pas fonctionné ? [...] Donc, je leur ai demandé ce qu'ils pensaient ce qui ne fonctionnait pas, et tout de suite ils ont dit que, d'après eux, c'est le capteur. J'ai dit : " Qu'est-ce que vous pourriez faire pour améliorer votre capteur? "Tout de suite, ils ne savaient pas quoi me répondre exactement, donc ils ont été tout de suite rechercher dans leur petit livre d'installation de capteur. Ils sont allés fouiller et ils ont réussi à installer le capteur de façon plus solide. " (E5, entrevue 2, avril 2018)

Ces exemples proposent des rétroactions axées sur l'autoévaluation et l'autorégulation. Cependant, d'autres types de rétroactions ont pu être constatés chez l'enseignante comme des rétroactions sur la tâche: «Qu'est-ce que tu veux que ton robot fasse vraiment? » II y a également eu des rétroactions sur le processus : 


\title{
8
}

\section{REVUE HYBRIDE DE L'ÉDUCATION}

\begin{abstract}
" Ici il te demande le diamètre. Là tu as le périmètre. Ici tu prends la distance. (Des fois la distance ils mettaient la même chose que le périmètre.) Est-ce que c'est logique que ta distance soit la même chose que ton périmètre ? Ils disent non et ils retournent à leur place. " (E5, entrevue 2, avril 2018)
\end{abstract}

La variété des types de rétroactions proposée par l'enseignante met de l'avant sa compréhension des objets d'apprentissage ciblés. Elle se centre sur les démarches que les élèves doivent utiliser. Aussi, en optant pour le questionnement, une démarche centrale en didactique de science et technologie, ses rétroactions se centrent naturellement sur les objets d'apprentissage.

\section{Focus group (décembre 2018) : intégrer la démarche de rétroaction à son quotidien}

La dernière rencontre a permis de faire un retour sur l'évolution des pratiques des enseignantes. Le premier constat a été de voir que les enseignantes avaient transféré leurs pratiques de rétroactions dans d'autres disciplines.

« L'an passé... Vous êtes venus nous voir pour nous demander les rétroactions qu'on faisait avec nos élèves. J'avais noté mes exemples de rétroactions que je faisais. J'avais vraiment fait l'exercice de mettre l'accent là-dessus en science. Là, je me suis rendu compte que ça l'avait déteint sur moi. J'en fais dans les autres matières maintenant. Juste hier, j'accueillais une stagiaire suisse qui venait voir ce qui se passait au 3 e cycle, parce qu'elle était au préscolaire-primaire. On jasait et elle est venue me voir en action pendant qu'ils étaient en production écrite. Là, on aurait pu penser que je serais assise à mon bureau, mais je circulais parce que les jeunes venaient à moi beaucoup. Est-ce que c'était correct? Je ne répondais jamais par oui ou non. Je ne validais pas leur réponse. J'étais toujours dans le questionnement, je répondais par des questions... j'avais pigé ça dans mes sciences. Donc je suis rendue comme ça et je pense que l'autonomie s'est développée plus depuis que je fais ça. " (E2, $3^{\text {e }}$ rencontre, décembre 2018) 


\section{$\&$}

\section{REVUE HYBRIDE DE L'ÉDUCATION}

Cette enseignante a pris conscience de l'importance des rétroactions pour accompagner l'élève à devenir autonome dans ses apprentissages, mais, aussi, pour l'accompagner à mieux comprendre ses propres stratégies. La rencontre a aussi permis de constater que les enseignantes ne cherchent plus la bonne réponse, mais plutôt à évaluer les démarches mises en œuvre par les élèves.

"Quand je fais ma robotique, je leur dis dès le départ: "Ce n'est pas parce que ton trajet est réussi que tu vas avoir une meilleure note que celui qui ne l'a pas réussi. " C'est vraiment dans la façon d'expliquer pourquoi ça fonctionne ou ça ne fonctionne pas que j'évalue. " (E5, $3^{\mathrm{e}}$ rencontre, décembre 2018)

Là encore, il y a eu un changement chez l'enseignante en ce qui a trait à sa compréhension de la discipline. En modifiant sa conception, en se centrant sur les critères et en les interprétant selon les démarches didactiques, cette enseignante s'est distanciée d'une démarche axée sur la bonne réponse. On s'approche ainsi beaucoup plus des visées des compétences en science et technologie.

\section{Discussion et conclusion}

Deux idées fortes émergent de cette analyse. La première fait ressortir l'arrimage entre l'activité didactique et l'activité d'évaluation et permet de faire un lien fort entre le questionnement et la rétroaction sur les apprentissages. La deuxième idée concerne l'importance de la recherche collaborative pour réfléchir aux pratiques, mais aussi pour développer des espaces de coconstruction de savoirs sur ces pratiques pour les praticiens et pour les chercheurs en éducation.

\section{Idée 1 : Le questionnement comme base à la rétroaction des apprentissages}

La réflexion portée sur l'évaluation des apprentissages, et plus particulièrement sur les critères d'évaluation, a amené les enseignantes à réorienter leur planification en science et technologie. En se concentrant sur les critères d'évaluation, elles ont mieux intégré les démarches d'investigation dans leur enseignement. Cet ajustement a modifié le choix des productions des élèves sur lesquelles elles posaient un regard évaluatif. Ainsi, au lieu d'opter pour un examen axé sur la mémorisation de connaissances précises, elles ont choisi la réalisation d'une entrevue avec l'élève, l'observation durant la démarche, ou l'élaboration de cartes conceptuelles pour évaluer les élèves. Comme les productions changent, les objets d'apprentissage évalués changent également. On passe de l'évaluation de connaissances à l'évaluation de la description d'un problème, de la démarche mise en œuvre par l'élève, de la maîtrise des 


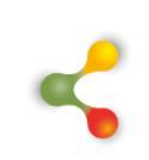

\section{REVUE HYBRIDE DE L'ÉDUCATION}

connaissances et des outils utilisés. Le jugement de l'enseignant est ainsi plus directement en lien avec les compétences visées dans le PFEQ (MEQ, 2001). L'arrimage des critères et des démarches d'investigation a permis de constater que lorsque les enseignantes utilisaient le questionnement pour relancer l'élève dans sa réflexion, elles étaient également en train de rétroagir auprès de ce dernier pour qu'il régule ses apprentissages. Et, comme les cibles d'apprentissage étaient plus claires et que les choix permettaient de mettre en place des démarches d'investigation, ces rétroactions devenaient plus pertinentes et cohérentes avec l'activité d'apprentissage. Au lieu de porter uniquement sur le résultat, ces rétroactions concernaient directement la tâche, le processus ou encore l'autoévaluation de l'élève. Cela nous amène à proposer une nouvelle figure (figure 2) qui présente le processus ayant mené à des rétroactions arrimées à l'activité didactique. 


\section{REVUE HYBRIDE DE L'ÉDUCATION}

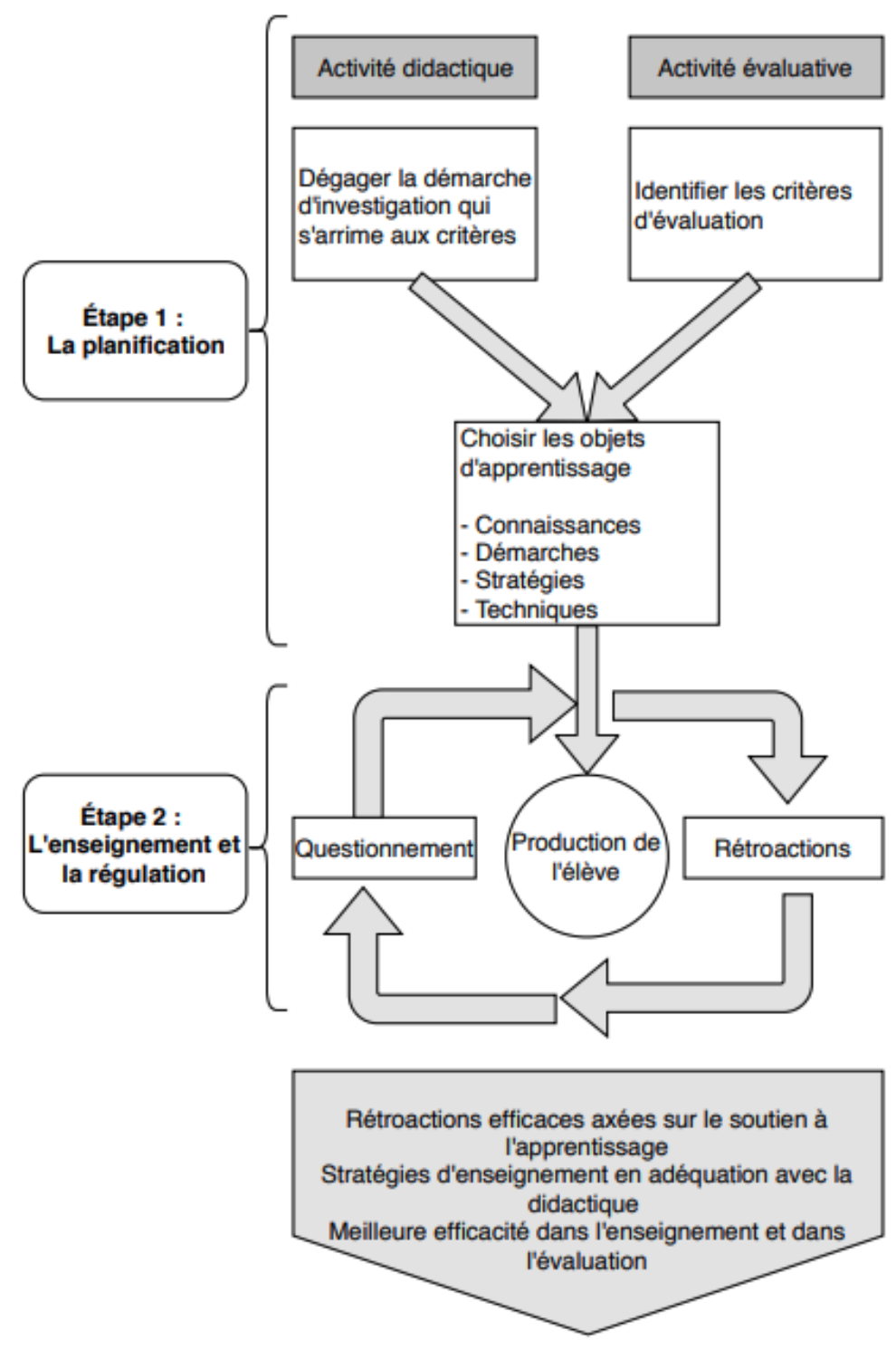

Figure 2 : Processus de mise en place de rétroactions axées sur l'activité didactique

Il y a donc deux étapes dans le processus, soit l'étape de planification, qui consiste à identifier les objets d'apprentissage en lien avec les démarches d'investigation et les critères d'évaluation. La deuxième étape consiste à l'activité d'enseignement et de régulation en classe. En général, cette activité propose une production de l'élève qui peut être la rédaction d'une question, d'une hypothèse, la proposition ou la réalisation 


\section{$\&$}

\section{REVUE HYBRIDE DE L'ÉDUCATION}

d'une démarche, l'utilisation d'outils, la compilation de données, l'explication d'un phénomène, la création d'un modèle ou d'un prototype. Pendant que l'élève réalise la tâche, l'enseignant questionne et rétroagit en regard des actions ou des réponses de l'élève. II peut ainsi soutenir l'apprentissage de l'élève, proposer des rétroactions précises qui permettent de réguler rapidement l'apprentissage, revoir ses stratégies d'enseignement, et finalement être plus cohérent dans son enseignement et dans son évaluation. Dans le cadre de cette recherche, ce processus a permis de réduire les inquiétudes des enseignantes par rapport à l'évaluation en science et technologie et de leur donner confiance dans leur enseignement de la science et technologie, ce qui nous amène à la deuxième idée émergeant de l'analyse des résultats.

\section{Idée 2 : La recherche collaborative comme espace de coconstruction de savoirs sur les pratiques}

Pour en arriver à un modèle qui arrime à la fois l'activité didactique et l'activité en évaluation, le premier temps a été de discuter les cadres de références mobilisés par les trois chercheures pour réfléchir à la rétroaction et à l'évaluation en science, technologie et univers social. L'arrimage entre les démarches d'investigation en science et technologie et les démarches d'évaluation pour soutenir l'apprentissage n'était, au premier abord, pas si évident. Le premier espace collaboratif a donc été celui entre chercheurs pour bien comprendre les processus sous-jacents à chacun de ces cadres issus de la didactique de la discipline et des travaux sur l'évaluation des apprentissages. Dans un deuxième temps, bien que théoriquement les visées d'arrimage étaient possibles, il était essentiel de voir si elles étaient viables dans les pratiques enseignantes. Le deuxième espace collaboratif, celui avec les enseignantes, était donc incontournable pour coconstruire avec elle un modèle qui permette d'arrimer à la fois l'activité didactique et l'activité évaluative. Les pratiques des enseignantes, saisies à travers leurs réflexions durant les rencontres et les entrevues, ont permis de raffiner le modèle. Le lien entre le questionnement et la rétroaction s'est fait en s'appuyant sur leurs pratiques de classe. Elles ont également questionné certains choix de productions, comme ceux de l'entrevue et de la carte conceptuelle qui, au premier abord, n'étaient pas des choix naturels pour évaluer les apprentissages en science et technologie. Finalement, elles ont confirmé qu'en ciblant les critères d'évaluation au départ, elles étaient en mesure de mieux orienter l'enseignement et de mieux soutenir l'apprentissage de l'élève en rétroagissant tout au long de l'activité.

II y a donc lieu de poursuivre la réflexion autour de l'évaluation des apprentissages. La problématique portait sur les stratégies pour accompagner l'apprentissage. Cet article a mis en évidence l'importance de travailler de concert entre didacticiens et spécialistes de l'évaluation des apprentissages pour mieux arrimer l'activité didactique et l'évaluation. Grâce à la collaboration et à l'implication des enseignantes, nous avons été en mesure de revoir nos cadres de références, de les ajuster et de les 


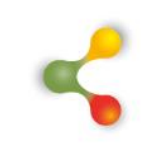

\section{REVUE HYBRIDE DE L'ÉDUCATION}

valider pour qu'ils soient viables en milieu scolaire. Le même constat a été réalisé pour l'enseignement de l'univers social (Monney, Duquette, Couture, 2020). L'arrimage avec d'autres disciplines scolaires reste à réaliser et pourrait faire l'objet de futures recherches en collaboration avec les principaux experts des milieux scolaires, les enseignants. 


\section{REVUE HYBRIDE DE L'ÉDUCATION}

\section{Références}

Akkari, A. et Barry, V. (2018). Pour une école inclusive : des intentions aux réalisations. Introduction. Revue internationale d'éducation de Sèvres, (78), 37-46. http://journals.openedition.org/ries/6394

Allal, L. et Lopez, L. M. (2007). Régulation des apprentissages: orientations conceptuelles pour la recherche et la pratique en éducation. Dans L. Allal et L. Mottier Lopez (dir.), Régulation des apprentissages en situation scolaire et en formation (p. 7-23). De Boeck.

Astolfi, J.-P., Peterfalvi, B. et Vérin, A. (1998). Comment les enfants apprennent les sciences. De Boeck.

Baribeau, A. (2009). Analyse des pratiques d'évaluation des compétences d'enseignants de français, secondaire premier cycle, pour établir un jugement professionnel lors du bilan des apprentissages [mémoire de maîtrise, université du Québec à Trois-Rivières]. Dépôt-e. http://depote.uqtr.ca/id/eprint/1187/

Bélair, L. et Dionne, É. (2009). Évaluation des apprentissages dans le contexte québécois : entre décisions politiques et pratiques en salle de classe. Mesure et évaluation en éducation, 32(3), 77-100.

Bellehumeur, P. et Painchaud, R. (2008). Pratiques évaluatives et tensions en contexte de changement d'objet d'évaluation. De connaissances à compétences. Dans L. Lafortune et L. Allal (dir.), Jugement professionnel en évaluation (p. 79-94). Presses de l'Université du Québec.

Black, P. et Wiliam, D. (1998). Inside the black box. Raising standards through classroom assessment. Phi Delta Kappan, 139-148.

Conseil supérieur de l'éducation. (2017). Pour une école riche de tous ses élèves: S'adapter à la diversité des élèves, de la maternelle à la $5 e$ année $d u$ secondaire. Gouvernement du Québec. https://www.cse.gouv.qc.ca/publications/ecole-riche-eleves-50-0500/

Coquidé, M., Fortin, C. et Rumelhard, G. (2009). L'investigation: fondements et démarches; intérêts et limites. Aster, 49(5), 51-78.

De Ketele, J.-M. (2010). Ne pas se tromper d'évaluation. Revue française de linguistique appliquée, XV, 25-37. http://www.cairn.info/revuefrancaise-de-linguistique-appliquee-2010-1-page25.htm\#anchor citation 


\section{REVUE HYBRIDE DE L'ÉDUCATION}

Deaudelin, C., Desjardins, J., Dezutter, O., Thomas, L., Corriveau, A., Lavoie, J., ... Hébert, M. (2007). L'évaluation formative en contexte de renouveau pédagogique au primaire: analyse des pratiques au service de la réussite. Nouveaux cahiers de la recherche en éducation, 10(1), 27-45.

Desgagné, S. (1997). Le concept de recherche collaborative: I'idée d'un rapprochement entre chercheurs universitaires et praticiens enseignants. Revue des sciences de l'éducation, 23(2), 371-393.

Diedhiou, S. B. M. (2013). Façons de faire l'évaluation formative d'enseignants de français sénégalais: une analyse de leurs savoirs pratiques en contexte d'effectifs pléthoriques au Lycée [mémoire de maîtrise, université de Montréal]. Papyrus. https://papyrus.bib.umontreal.ca/xmlui/handle/1866/10862;jsessionid =F6D53B50D7865770B81C1B097B446CFA

Éthier, M. A. et Dalongeville, A. (2005). Du faux débat entre compétences et connaissances. Traces, 45(3), 14-16.

Hadji, C. (2015). L'évaluation à l'école. Paris : Nathan.

Issaieva, É. et Crahay, M. (2010). Conceptions de l'évaluation scolaire des élèves et des enseignants: validation d'échelles et étude de leurs relations. Mesure et évaluation en éducation, 33(1), 31-61.

Jorro, A. et Mercier-Brunel, Y. (2011). Les gestes évaluatifs de l'enseignant dans une tâche de correction collective. Mesure et évaluation en éducation, 34(3), 27-50. http://id.erudit.org/iderudit/1024794ar

Laurier, M. (2014). La politique québécoise d'évaluation des apprentissages et les pratiques évaluatives. Éducation et francophonie, 42(3), 31-49.

Laveault, D. (2014). Les politiques d'évaluation en éducation. Et après? Éducation et francophonie, 42(3). doi:10.7202/1027402ar

Leclerc, M.-J. (2015). L'évaluation, un élément crucial du processus d'apprentissage. Université de Montréal. http://rire.ctreq.qc.ca/wpcontent/uploads/2015/06/Marie-Josee-Leclerc-Methodesdevaluation.pdf

L'Écuyer, R. (1990). Méthodologie de l'analyse développementale de contenu. Presses de l'Université de Québec. 


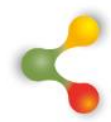

\section{REVUE HYBRIDE DE L'ÉDUCATION}

Lederman, N. G. et Lederman, J. S. (2012). Nature of Scientific Knowledge and Scientific Inquiry: Building Instructional Capacity Through Professional Development. Dans B. Fraser, K. Tobin et C. McRobbie (dir.), Second International Handbook of Science Education (vol. 1., p. 335-339). Springer.

Ministère de l'Éducation, du Loisir et du Sport. (2009). La progression des apprentissages au primaire. Gouvernement du Québec. http://www.education.gouv.qc.ca/enseignants/pfeq/primaire/

Ministère de l'Éducation, du Loisir et du Sport. (2011). Cadre d'évaluation des apprentissages. Gouvernement du Québec. http://www.education.gouv.qc.ca/fileadmin/site web/documents/educ ation/jeunes/pfeq/CE PFEQ science-technologie-primaire.pdf

Ministère de l'Éducation du Québec. (2001). Programme de formation de l'école québécoise. Gouvernement du Québec. http://www.meq.gouv.qc.ca/lancement/prog formation/index.htm

Monney, N., Duquette, C., Couture,C., Boulay, H. (2020), Réfléchir autour des considérations didactiques de l'univers social pour favoriser des pratiques évaluatives plus inclusives: quoi, pourquoi et comment? Revista Educativa 23.

Morrissette, J. (2009). Une resocialisation de l'évaluation des apprentissages. Revue canadienne des jeunes chercheures et chercheurs en éducation, 1(2).

Morrissette, J. et Legendre, M.-F. (2011). L'évaluation des compétences en contexte scolaire: des pratiques négociées. Education Sciences \& Society, 2(2),

$120-132$

https://riviste.unimc.it/index.php/es s/article/viewFile/189/117

Mottier Lopez, L. et Cattafi, F. (2008). Le processus du jugement professionnel comme fil conducteur dans l'attribution des notes. Dans L. Lafortune et L. Allal (dir.), Jugement professionnel en évaluation ( $p$. 159-186). Presses de l'Université du Québec.

Ramel, S. et Vienneau, R. (2016). Des fondements sociologiques de l'inclusion scolaire aux injonctions internationales. De Boeck Supérieur.

Savoie-Zajc, L. (2011). La recherche qualitative/interprétative en éducation. Dans T. Karsenti et L. Savoie-Zajc (dir.), La recherche en éducation : étapes et approches. ( $2^{\mathrm{e}}$ éd., p. 123-150). Édition du CRP. 


\section{REVUE HYBRIDE DE L'ÉDUCATION}

Scallon, G. (2004). L'évaluation des apprentissages dans une approche par compétences. Édition du renouveau pédagogique.

Scriven, M. (1967). The methodology of evaluation. Dans R. W. Tyler, R. M. Gagné et M. Scriven (dir.), Perspectives of curriculum evaluation (p. 39-83). Rand McNally.

Seixas, P. (2005). Historical Consciousness : the Progress of Knowledge in a Postprogressive Age. Dans J. Straub (dir.), Narration, Identity and Historical Consciousness (p. 141-162). Berghahn Books.

Talbot, L. et Arrieu-Mutel, A. (2012). Décrire, comprendre et expliquer les pratiques d'enseignement d'un professeur de lycée. Education \& didactique, 6(3), 65-95. doi:10.4000/educationdidactique.1504

UNESCO. (2016). Global education monitoring report, 2016: Place: inclusive and sustainable cities. https://unesdoc.unesco.org/ark:/48223/pf0000246230

Van der Maren, J.-M. (1995). Méthodes de recherche pour l'éducation. Les Presses de l'Université de Montréal.

Wolfs, J.-L. (2008). Analyser des pratiques éducatives visant à faire participer l'apprenant à l'évaluation diagnostique, au pilotage et à la régulation de ses apprentissages. Dans J. Grégoire (dir.), Evaluer les apprentissages : Les apports de la psychologie cognitive (p. 175-186). De Boeck. 